Ophthalmologe 2013 · 110:298

DOI 10.1007/s00347-012-2709-6

Online publiziert: 9. März 2013

(c) Springer-Verlag Berlin Heidelberg 2013

\section{T. Dietlein}

Zentrum für Augenheilkunde der Universität Köln

\title{
Aktuelle Glaukomchirurgie
}

Viskokanaloplastik, Trabektom und neue Mikrostents haben in den letzten Jahren die Diskussion rund um die aktuellen Entwicklungen in der Glaukomchirurgie beherrscht. Diese Verfahren rütteln bei vielen Kongressen und Glaukomtagungen am Thron des Standardverfahrens, der Trabekulektomie. Das über Jahrzehnte ausgereifte und verfeinerte Verfahren der Trabekulektomie ist allerdings nicht ohne Grund weiterhin die Nummer eins bei den inzisionalen glaukomchirurgischen Eingriffen in Deutschland.

\section{D) Die Trabekulektomie ist Standardverfahren bei inzisionalen glaukom- chirurgischen Eingriffen}

Klink und Grehn präsentieren hier einen aktuellen und sehr wichtigen Überblick zur Trabekulektomie und ihren zeitgemäßen technischen Modifikationen. Die überzeugenden Argumente für die Trabekulektomie bleiben bis dato die lange Wirksamkeit und die gute Senkung des Augeninnendruckniveaus inklusive der Augeninnendruckfluktuationen.

Ein viel diskutiertes Thema unter $\mathrm{Au}$ genärzten war in den letzten Jahren auch die häufig propagierte Frühbehandlung des Pigmentglaukoms durch die Laseriridotomie. Nach anfänglich großer Begeisterung für diesen Therapieansatz in den 1990er-Jahren fehlte es in den darauffolgenden Jahren an verlässlichen Belegen eines funktionellen Nutzens für den Patienten. Im klinischen Alltag wird dieser "prophylaktische" Eingriff immer noch sehr vielen Patienten aller Altersklassen mit Pigmentdispersionssyndrom bis hin zum fortgeschrittenen Pigmentglaukom empfohlen. Rosentreter et al. stellen in ihrem Beitrag klar, dass eine solche sehr breite Empfehlung zur Laseriridotomie nach Durchsicht der Literatur nicht sinnvoll erscheint.

Eine ganze Reihe schwieriger Fragen stellt sich bei Glaukompatienten, die sich einer Kataraktoperation unterziehen oder kombiniert operiert werden sollen. Oft mit Prostaglandinderivaten vorbehandelt, ist es bei diesen Glaukompatienten strittig, ob ein Absetzen dieser topischen Antiglaukomatosagruppe vor der Kataraktoperation notwendig ist, um das Risiko eines postoperativen Makulaödems niedrig zu halten. Auch die Wahl der Intraokularlinse beim Glaukompatienten ist durchaus sehr komplex. Bis heute nicht umfassend und valide untersucht sind die Auswirkungen der unterschiedlichen „Premium-Intraokularlinsen“ auf das schon gestörte Kontrastsehen des Glaukompatienten.

Sehr unterschiedlich sind zuweilen die Einschätzungen der Operateure, ob zunächst eine alleinige Kataraktoperation bei medikamentös nicht reguliertem Augendruck versucht werden sollte oder ob es klüger ist, den augendrucksenkenden Eingriff (Trabekulektomie) vorzuziehen mit der eindeutigen Hypothek des späteren Zweiteingriffs (Kataraktoperation). Hier sind kammerwinkelchirurgische Glaukomeingriffe in Kombination mit der Kataraktchirurgie häufig ein risikoarmer Kompromiss für den Patienten, auch wenn das drucksenkende Potenzial natürlich nicht mit der Trabekulektomie vergleichbar ist. Das kombinierte Vorgehen beim älteren Glaukompatienten zielt ganz besonders auf die Verbesserung der Lebensqualität ab, einerseits in der Hoffnung einer zentralen Visusverbesserung, andererseits in der Hoffnung, die Anzahl der chirurgischen Eingriffe für den Patienten zu reduzieren.
In unseren beiden Beiträgen zu dieser Thematik möchten wir zumindest auf einen Teil dieser komplexen Fragen eingehen und eine informative Übersicht über die mittlerweile gängigen Formen der kombinierten Glaukom-Katarakt-Chirurgie geben.

Allen Autoren danke ich nochmals herzlich für das sehr konstruktive Zusammenwirken. Wir alle hoffen, Ihnen mit unseren Beiträgen neue hilfreiche Anregungen für die klinisch-chirurgische Versorgung Ihrer Glaukompatienten zu geben.

Ihr

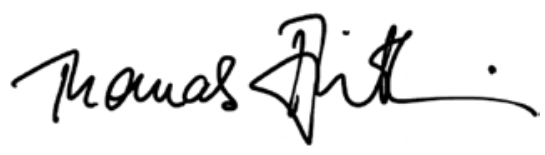

Thomas Dietlein

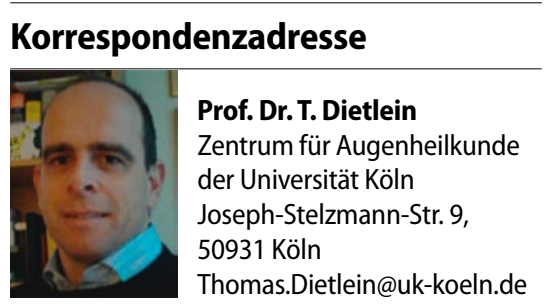

